\title{
Anomalies as a signature of extra dimensions
}

\author{
Alexey Boyarsky*, Oleg Ruchayskiył Mikhail Shaposhnikov*.
}

\begin{abstract}
The anomaly cancellation condition of the Standard Model may be unnatural in theories with extra dimensions as an anomaly of a low-energy 4-dimensional theory can be canceled by an inflow from a bulk. This inflow may give rise to an observable effect at low energies. We analyze several physical models in which this effect exists and estimate constraints on its value, imposed by the modern experimental data. We show that the effect can be large enough to be observed even when these constraints are satisfied. Positive result of such an experiment would be a low-energy signature of the existence of extra dimensions.
\end{abstract}

\section{Introduction}

Models with extra dimensions are interesting candidates to describe physics beyond the Standard Model (SM). It is generally assumed that such a new physics appears at high energies, say at $1 \mathrm{TeV}$ or above. It is natural to ask whether there could exist some low energy signatures of extra dimensions. In this paper we will show, that in addition to a possible modification of gravitation Newton's law at distances of a fraction of millimeter [1], there could be quantum effects, related to the anomalies of chiral gauge theories. Anomalies do not depend on a scale (e.g. the scale of the extra dimensions) which makes possible that corresponding effects are visible at low energy.

In the Standard Model in 4 dimensions the fermion couplings are chiral, and the hypercharges of quarks and leptons are chosen in such a way as to ensure cancellation of all gauge anomalies [2]. However, in theories with extra dimensions, where the SM fields are realized as zero modes of a higher-dimensional ones, only the full (higher-dimensional) theory should be anomaly free and there is no reason to expect separate anomaly cancellation for the brane fields (for an explicit example of a consistent brane-world theory with an anomaly on the brane, see [3]). The aim of this paper is to study exactly this type of models.

Qualitatively, if the theory on the brane is anomalous, then there is a specific type of brane-bulk interaction - anomaly inflow [4]. It is described by Chern-Simons-like terms in

${ }^{*}$ Ecole Polytechnique Fédérale de Lausanne, Institute of Theoretical Physics, FSB/ITP/LPPC, BSP 720, CH-1015, Lausanne, Switzerland

†Institut des Hautes Études Scientifiques, Bures-sur-Yvette, F-91440, France 
the low-energy effective action in the bulk. These terms are not gauge invariant in the presence of a brane. Therefore, they generate currents, flowing to the brane and ensuring the gauge invariance of the full theory. Such a mechanism appears in many problems: in field theories with solitonic backgrounds (see e.g. [5, 3]), in string and M-theory [6].

What would be the consequences of the presence of inflow currents from the point of view of a low energy physics on a brane? For example, can one distinguish between a 4-dimensional anomaly-free theory and a theory with extra dimensions and anomaly inflow?

Naively, one would think that anomaly inflow current is a flux of particles from higher dimensions. From 4-dimensional point of view such a process would seem as a "loss of unitarity". However, for a brane-world model to be phenomenologically acceptable, there should be a mass gap between zero and bulk modes of matter fields and zero mode particles cannot leave the brane at energies below the mass gap. Instead, the inflow current by its nature is a vacuum or non-dissipative current. Although inflow current is not carried by real particles, the redistribution of the charges in the Dirac sea of the full (5-dimensional) theory leads to an appearance of an electric charge on the brane [7]. As a result, for example, electric field, created by a capacitor, changes when the latter is placed in a magnetic field. In particular, a field appears outside the capacitor, while inside it diminishes in value and gets redistributed in space. Another example is a charged elementary particle: when placed in a magnetic field its electric charge gets screened. From the 4-dimensional point of view it looks as if photon had acquired a mass, depending on a magnetic field. In addition, particle with both electric charge and magnetic moment acquires time-dependent dipole moment. These effects would not be present in a purely 4-dimensional theory with anomaly canceled by addition of new (chiral) particles at higher energies, and thus can be considered a signature of extra dimension. As we will see, they are not suppressed by the mass scale of extra dimensions.

In the present paper we study the physical manifestations of the above effects and suggest an experiment in which they can be observed. ${ }^{1}$ The paper is organized as follows. We describe the observable effects of anomaly inflow in Section 2 and then turn in Section 3 to the question of possible "anomalous" extensions of SM. Next, we discuss an experiment in which the screening of electric charge can be observed. In Section 4 we analyze several physical models and obtain quantitative estimates of the screening.

\section{Anomalous electrodynamics and its observational signatures.}

\subsection{5 -dimensional electrodynamics on a brane}

To demonstrate desired effects we start with a $U(1)$ theory in 5 dimensions. This model was discussed in details in [7], here we only remind its main features. We consider a 5-dimensional

\footnotetext{
${ }^{1}$ We analyze only gauge anomalies here. Brane-world models with global chiral anomaly has been studied in [8]. They may lead to the solution of the strong CP problem.
} 
brane-world which has both chiral fermions and gauge fields localized on a brane. The low energy effective action of this theory consists of three parts: kinetic term for the gauge fields; Chern-Simons like term, describing a brane-bulk interaction, leading to inflow; and an action of (chiral) fermionic zero modes, localized on the brane (hence $\delta(z)$ in the last term) $:^{2,3}$

$$
S=-\frac{1}{4 \mathbf{e}^{2}} \int d^{5} x \Delta(z) F_{a b}^{2}+\frac{1}{4} \int d^{5} x \kappa(z) \epsilon^{a b c d e} A_{a} F_{b c} F_{d e}+\int d^{5} x \mathcal{L}_{\mathrm{z} \cdot \mathrm{m}} \delta(z) .
$$

Here $\mathbf{e}$ is a five-dimensional charge, with the dimensionality of (length) $)^{\frac{1}{2}}$. Localization on chiral fermions is achieved as in e.g. [9]. To localize $U(1)$ gauge field we modified kinetic term in (2.1): a $U(1)$ gauge field will possess a massless zero mode on the brane, if the factor $\Delta(z)$ in the kinetic term is chosen properly (we refer for details to $[10,11,12,13,7,14]$ ).

The charges in the full theory are chosen such that the theory of fermion zero modes (described by $\mathcal{L}_{\text {z.m }}$ ) suffers from a gauge anomaly. Namely, the electric current of these modes $j_{\text {z.m }}^{\mu} \equiv \frac{\delta \mathcal{L}_{\text {z.m }}}{\delta A_{\mu}}$ is not conserved:

$$
\partial_{\mu} j_{\mathrm{z} \cdot \mathrm{m}}^{\mu}=\frac{3}{2} \kappa_{0} \epsilon^{\mu \nu \lambda \rho} F_{\mu \nu} F_{\lambda \rho} \delta(z)
$$

Parameter $\kappa_{0}$ is proportional to the difference of the (cubes) of the charges of left and right-handed fermions [15, 7]. From a 4-dimensional point of view such a theory would be inconsistent at quantum level [16]. However, being embedded in a 5-dimensional theory (2.1), it becomes perfectly well-defined. This is due to the special type of an interaction (anomaly inflow [4]) represented by a Chern-Simons-like term in (2.1). Compared to the usual cases when Chern-Simons terms appeared in an effective action [17], this term has an additional factor $\kappa(z)$. In the limit of an infinitely thin wall and the large fermionic mass gap it reduces to $\kappa(z)=\kappa_{0} \operatorname{sign}(z)$. Due to the presence of the brane, which plays the role of a boundary, Chern-Simons term is not gauge invariant and its variation cancels that of $\mathcal{L}_{\text {z.m. }}$. Namely, the divergence of the Chern-Simons current is opposite to that of $j_{\mathrm{z} . \mathrm{m}}^{\mu}$ :

$$
\partial_{\mu} j_{\mathrm{z} \cdot \mathrm{m}}^{\mu}=-\partial_{a} J_{\mathrm{CS}}^{a} \neq 0
$$

where the inflow current $J_{\mathrm{CS}}$ is defined via ${ }^{4}$

$$
J_{\mathrm{CS}}^{a}\left(x^{a}\right)=\frac{\delta S_{\mathrm{CS}}}{\delta A_{a}}=\frac{3}{4} \kappa(z) \epsilon^{a b c d e} F_{b c}\left(x^{a}\right) F_{d e}\left(x^{a}\right)+\frac{1}{2} \kappa^{\prime}(z) \delta_{\mu}^{a} \epsilon^{\mu \nu \lambda \rho} A_{\nu} F_{\lambda \rho}, \quad \kappa^{\prime}(z)=\frac{d \kappa(z)}{d z} .
$$

If the theory were purely four-dimensional, non-zero divergence (2.2) of the electric current $j_{\mathrm{z} . \mathrm{m}}^{\mu}$ would have described the rate of anomalous particle production, essentially the

\footnotetext{
${ }^{2}$ Our conventions are as follows: Latin indices $a, \ldots, e=0, \ldots, 4$, Greek $\mu, \nu=0, \ldots, 3$. We are using mostly negative metric. Our brane is stretched in $0, \ldots, 3$ directions and is located at $z=0$ (where $z \equiv$ $\left.x^{4}\right)$. We will use notations $t, x$ for $x^{0}, x^{1}$ and choose polar coordinates $(r, \theta)$ in the plane $\left(x^{2}, x^{3}\right)$. Spatial coordinates on the brane $\left(x^{1}, x^{2}, x^{3}\right)$ will somtimes be denoted by $\vec{x}$.

${ }^{3}$ We choose all the characteristic energy scales in the problem to be much less than the mass gap of the fermionic zero modes $m_{\psi}$, therefore their profile can be approximated by the delta-function $\delta(z) \approx$ $2 m_{\psi} e^{-m_{\psi}|z|}$, which appears in eqs. (2.1), (2.2), etc.

${ }^{4}$ The term in (2.4), proportional to the $\kappa^{\prime}(z)$, can be thought of as a local counterterm, making the current of zero mode $j_{\mathrm{z} . \mathrm{m}}^{\mu}$ anomalous. See $[18,5]$.
} 
measure of non-unitarity of the theory. In the 5-dimensional brane-world this divergence gets canceled by that of the Chern-Simons (or inflow) current $J_{\mathrm{CS}}^{a}$ (eq. (2.3)). According to the definition (2.4) for configurations of 4-dimensional electro-magnetic fields, such that

$\epsilon^{\mu \nu \lambda \rho} F_{\mu \nu} F_{\lambda \rho} \neq 0$, there exists the component $J_{\mathrm{CS}}^{z}$, i.e the Chern-Simons current "flows onto the brane". However, it would be wrong to think about this current as "bringing to the brane particles from extra dimension". In reality this is a vacuum current - a collective effect of redistribution of the bulk (massive) fermions. At the energies of interest these fermions cannot be excited from the vacuum and cannot create actual charge carriers. The effect of the redistribution of the particles in the vacuum, however, does not depend on their mass and thus inflow interaction is not suppressed by the mass gap. (This fact can be checked by the direct microscopic computations of the vacuum average of $\left\langle 0\left|J_{\mathrm{CS}}^{z}\right| 0\right\rangle$ in the full theory). One may think of the effect of the vacuum current as of a dielectric susceptibility of the vacuum of four-dimensional theory, embedded in the 5-dimensional space-time.

We see that the main characteristic feature of theories with anomaly on the brane, is the presence of the vacuum inflow current from extra dimensions. The presence of such a current exhibits itself at low energies, as we discuss below.

\subsection{Anomalous electric field of a capacitor}

To design a setup in which anomaly inflow could exhibit itself we need a configuration of (five-dimensional) gauge fields in which $\left.\epsilon^{\mu \nu \lambda \rho} F_{\mu \nu}\left(x^{a}\right) F_{\lambda \rho}\left(x^{a}\right)\right|_{z=0} \sim \vec{E} \cdot \vec{H}$ is non-zero (we will take $F^{01}=E$ and $F^{23}=H$ in suitably chosen coordinate system). We realize such fields by two parallel infinite plates in the vacuum, separated in the direction $x$ by the distance $2 d-\mathrm{a}$ capacitor with initial charge densities $\pm \sigma_{0}$ on plates, placed in the magnetic field. Magnetic field is created by an (infinite in the $x$-direction) solenoid with the radius $R$. Below we report the results, for details a reader is referred to [7].

A final stage of anomaly inflow in this system is such that the static solution (nonperturbative in $\kappa_{0}$ ) will form. The main property of this solution is that an electric field gets screened as if photon had become massive with the mass

$$
m_{0}^{2} \sim \kappa_{0} \boldsymbol{H}_{0}\left(\mathbf{e}^{2} M\right)=\kappa_{0} e^{2} \boldsymbol{H}_{0}
$$

(where $e^{2}$ is a 4 -dimensional coupling constant, related to the 5 -dimensional $\mathrm{e}^{2}$ via $e^{2} \sim$ $\left.\mathbf{e}^{2} M\right)$. The mass $m_{0}$ depends only on the 4-dimensional quantities (fine-structure constant $e^{2}$ and the magnetic field $\boldsymbol{H}_{0}$ as seen by an observer on a brane). Notice, that $m_{0} \ll M$ for any physically plausible values of magnetic field $\boldsymbol{H}_{0} \cdot{ }^{5}$

The profile of a 4-dimensional electric field in the static state is given by

$$
\boldsymbol{E}^{x}(x)=-\frac{\boldsymbol{E}_{0}}{2}\left[\operatorname{sign}(x-d) e^{-m_{0}|x-d|}-\operatorname{sign}(x+d) e^{-m_{0}|x+d|}\right] .
$$

Here $\boldsymbol{E}_{0}$ is a value of 4 -dimensional field, which would be created between the plates of the capacitor in the theory without anomaly $\boldsymbol{E}_{0}=\sigma_{0} e^{2}$. The characteristic time $\tau_{0}$ over which

\footnotetext{
${ }^{5}$ Recall that 1 Gauss $=1.95 \cdot 10^{-20} \mathrm{GeV}^{2}$ while $M \gtrsim 10^{4} \mathrm{GeV}$. For the maximal values of magnetic fields, see Section 4.
} 
the solution (2.6) is established is of the order $m_{0}^{-1}$. Of course, as $m_{0} \rightarrow 0$ (2.6) reproduces an electric field of an infinite capacitor. The full (5-dimensional) electrostatic potential, corresponding to the solution (2.6) has the following form:

$$
\Phi(x, z)=\phi_{0}(x) \chi_{0}(z)=-\frac{\mathbf{e}^{2} \sigma_{0}}{2 m_{0}}\left(e^{-m_{0}|x-d|}-e^{-m_{0}|x+d|}\right) \chi_{0}(z)
$$

with the $x$ profile $\phi_{0}(x)$ satisfying Poisson equation with mass $m_{0}: \partial_{x}^{2} \phi_{0}(x)-m_{0}^{2} \phi_{0}(x)=$ $\sigma_{0}(\delta(x-d)+\delta(x+d))$.

The profile of the solution (2.7) in the $z$ direction $\chi_{0}(z)$ is sharply localized in the region ${ }^{6}$

$$
|z| \lesssim \frac{1}{M} \log \frac{M}{m_{0}} \approx \frac{1}{2 M} \log \frac{M^{2}}{e^{2} \kappa_{0} \boldsymbol{H}_{0}} .
$$

Thus, the function $\Phi(x, z)$ exponentially decays in $z$ direction on the scale proportional to the $M^{-1}$ and depending on $\kappa_{0}$ (potentially very small) only logarithmically, while the scale $m_{0}^{-1}$ of the exponential decay of the potential in the $x$ direction does not depend on $M$ and is proportional to the $\sqrt{\kappa_{0}}$.

To summarize: an effect of anomaly inflow exhibits itself via a change of a distribution of an electric field in a capacitor. The field diminishes inside

$$
\boldsymbol{E}_{\text {inside }}^{x}=\boldsymbol{E}_{0} e^{-m_{0} d} \cosh m_{0} x, \quad|x|<d,
$$

but appears outside:

$$
\boldsymbol{E}_{\text {outside }}^{x}=-\boldsymbol{E}_{0} e^{-m_{0}(|x|-d)}\left(1-e^{-2 m d}\right), \quad|x|>d .
$$

If $\kappa_{0}$ is such that $m_{0} d \ll 1$, the field outside the capacitor (for $\frac{1}{M} \ll(|x|-d) \ll \frac{1}{m_{0}}$ ) is almost constant, given by

$$
\boldsymbol{E}_{\text {outside }}^{x} \approx 2 \boldsymbol{E}_{0}\left(m_{0} d\right)
$$

\section{The Standard Model with anomaly inflow}

In this section we apply the logic of Section 2 to SM. Indeed, if the SM fields are localized on a brane in a 5-dimensional world, there is no apparent reason to expect a separate anomaly cancellation for them. For simplicity we consider the electroweak $S U(2) \times U(1)$ theory with only one generation of fermions (an addition of extra generations does not change the analysis). The action for the $S U(2)$ and $U(1)$ gauge fields is similar to that in eq. (2.1). The warp-factor $\Delta(z)$ is such that there is a zero mode, localized on the brane. We also add a Higgs $\phi$ field, which is an $S U(2)$ doublet. Its mass $m_{\phi}^{2}(z)$ is negative at $z=0$ and tends to

\footnotetext{
${ }^{6}$ In case of the warp factor $\Delta(z)=\exp (-2 M|z|)$ [7] the profile function $\chi_{0}(z)$ is given by $\frac{m_{0} e^{M|z|}}{\sqrt{6 \pi}} K_{\nu}\left(\frac{m_{0}^{2}}{4 M^{2}} e^{2 M|z|}\right)$ where $K_{\nu}$ is the modified Bessel function of the second kind with the index $\nu=\frac{1}{2} \sqrt{1-m_{0}^{2} / M^{2}}$ (we assume that $m_{0}<M$ ). Therefore outside the region (2.8) potential $\Phi$ decays as an exponent $e^{-\Delta(z)}$. Notice, that $K_{\nu}$ is non-perturbative as a function of $\kappa_{0}$.
} 


\begin{tabular}{|c|c|c|c|c|c|c|}
\hline Particle: & $e_{L}$ & $e_{R}$ & $\nu_{L}$ & $Q$ & $u$ & $d$ \\
\hline Hypercharge $Y:$ & $-1+\kappa_{l}$ & $-2+\kappa_{l}$ & $-1+\kappa_{l}$ & $\frac{1}{3}+\kappa_{q}$ & $\frac{4}{3}+\kappa_{q}$ & $-\frac{2}{3}+\kappa_{q}$ \\
\hline
\end{tabular}$++$\begin{tabular}{|c|}
\hline$\nu_{R}$ \\
\hline$\kappa_{l}$ \\
\hline
\end{tabular}

Table 1: Modification of hypercharge assignments in SM. Standard assignments are shifted by $\kappa_{l}$ in lepton sector and by $\kappa_{q}$ in quark sector. The right-handed neutrino can be added to cancel also gravitational anomaly, but does not affect our analysis otherwise.

the positive constant in the bulk as $|z| \rightarrow \infty$. Therefore its VEV has a non-trivial profile $\langle\phi(z)\rangle$, with $\langle\phi(0)\rangle \neq 0$ and $\langle\phi(z)\rangle \rightarrow 0$ at $z \rightarrow \pm \infty$. So the $S U(2) \times U(1)$ symmetry is broken down to $U(1)_{\mathrm{EM}}$ on the brane and restored far from it. As a result, the vector boson acquires mass $\mathbf{m}_{\mathrm{z}}$ on the brane, while remains massless at large $|z|$.

We add fermions in the bulk, charged with respect to $S U(2) \times U(1)$. Fermionic zero modes are localized on the brane. There is a Yukawa interaction of these modes with the Higgs field $\phi$ :

$$
\mathcal{L}_{Y u k a w a}=\lambda_{e} \bar{E}_{L}^{a} \phi^{a} e_{R}+\lambda_{d} \bar{Q}_{L}^{a} \phi^{a} d_{R}+\lambda_{u} \epsilon_{a b} \bar{Q}_{L}^{a}\left(\phi^{+}\right)^{b} u_{R}+\lambda_{\nu} \bar{E}_{L}^{a} \epsilon_{a b}\left(\phi^{+}\right)^{b} \nu_{R}
$$

which leads to mass terms of brane fermions after spontaneous symmetry breaking. ${ }^{7}$ We can also add a right-handed neutrino $\nu_{R}$ with a Majorana mass term. The charges of these particles with respect to the $U(1)_{\text {Y }}$ group (hypercharges) are related due to the structure of the interaction (3.1):

$$
\left\{\begin{array}{l}
Y_{E}=Y_{\phi}+Y_{e}, \\
Y_{Q}=Y_{\phi}+Y_{d}, \\
Y_{Q}=Y_{u}-Y_{\phi} .
\end{array}\right.
$$

Hypercharges and isospins (charges with respect to the $S U(2)$ ) of particles are related to their electric charges via

$$
Q_{\mathrm{EM}}=\frac{1}{2} \sigma^{3}+\frac{Y}{2 Y_{\phi}}
$$

(here $\sigma^{3}$ is a Pauli matrix). We will choose the normalization $Y_{\phi}=1$ from now on.

Notice, that for any choice of hypercharges, consistent with (3.2), electrodynamics remains vector-like. One could (erroneously) conclude that we cannot expect to detect any consequences of anomaly inflow at low energies, as only electromagnetic background fields can be created, while the corresponding current is non-anomalous. However, we will show in the following sections that this is not the case, and in fact we can observe effect, identical to that of the Section 2.2.

\subsection{Charge difference of electron and proton and anomalies}

We consider the extension of SM in which one chooses arbitrary values of hypercharges, consistent with (3.2). This extension can be described in terms of two parameters $\kappa_{l}$ and $\kappa_{q}$, which shift the standard values of hypercharges in lepton and quark sectors correspondingly

\footnotetext{
${ }^{7}$ We use here the standard notations: left-handed fermions $\left(\nu_{L}, e_{L}, u_{L}, d_{L}\right)$ form a doublet with respect to the $S U(2)$ group, right-handed particles $\left(e_{R}, u_{R}, d_{R}\right)$ are singlets.
} 
(see Table 1). We are going to show that all anomalies, which arise as a result of our choice of hypercharges, will be proportional to the same combination of $\kappa_{l}$ and $\kappa_{q}$, which is equal to the charge difference between the electron and the proton.

For generic values of $\kappa_{l}$ and $\kappa_{q}$ in Table 1 the $U(1)_{Y}$ hyper-current $j_{\mathrm{Y}}^{\mu}$ becomes anomalous ${ }^{8}$

$$
\partial_{\mu} j_{\mathrm{Y}}^{\mu}=\frac{\operatorname{Tr}\left[Y^{3}\right]}{16 \pi^{2}} \epsilon^{\mu \nu \lambda \rho} \mathcal{F}_{\mu \nu} \mathcal{F}_{\lambda \rho}+\frac{\operatorname{Tr}\left[Y_{L}\right]}{16 \pi^{2}} \epsilon^{\mu \nu \lambda \rho} \operatorname{Tr} G_{\mu \nu} G_{\lambda \rho}
$$

Here $\mathcal{F}_{\mu \nu}$ is a $U(1)_{\mathrm{Y}}$ field strength of the $U(1)$ field $B_{\mu}$ (hyper-photon): $\mathcal{F}_{\mu \nu}=\partial_{\mu} B_{\nu}-\partial_{\nu} B_{\mu}$; $G_{\mu \nu}^{\alpha}$ is an $S U(2)$ non-Abelian field strength, $g^{\prime}$ and $g$ are $U(1)_{\mathrm{Y}}$ and $S U(2)$ coupling constants correspondingly. The first term in (3.4) comes from the triangular diagram with three $U(1)$ vertices and is proportional to the sum of cubes of hypercharges of all particles. The second term comes from the diagram with one $U(1)_{\mathrm{Y}}$ and two $S U(2)$ vertices. It is proportional to the sum of hypercharges of all left particles - those, having non-trivial $S U(2)$ charges. Traces in (3.4) are given by

$$
\operatorname{Tr}\left[Y^{3}\right]=6\left(\kappa_{l}+3 \kappa_{q}\right) \quad \text { and } \quad \operatorname{Tr}\left[Y_{L}\right]=-2\left(\kappa_{l}+3 \kappa_{q}\right) .
$$

Along with anomaly (3.4) there is also a non-conservation of $S U(2)$ current in the background $U(1)$ and $S U(2)$ fields (its coefficient is proportional to the same diagram as the second term in $(3.4))$ :

$$
D^{\mu} j_{\mu}^{\alpha}=\frac{\operatorname{Tr}\left[Y_{L}\right]}{8 \pi^{2}} \epsilon^{\mu \nu \lambda \rho} G_{\mu \nu}^{\alpha} F_{\lambda \rho}
$$

We see, that in our extension of the electroweak theory all anomalies are proportional to the same combination of $\kappa_{l}$ and $\kappa_{q}$ :

$$
\kappa \equiv \kappa_{l}+3 \kappa_{q}
$$

which we will call anomaly coefficient in what follows. In the absence of right-handed neutrino, the requirement of cancellation of both anomalies (3.4) and (3.6) would lead to the standard hypercharge assignments of SM (namely, $\kappa_{l}=\kappa_{q}=0$ ). With the right-handed neutrino being present, requirement of anomaly cancellation would only fix $\kappa=0$, and thus impose $\kappa_{l}=-3 \kappa_{q}$ condition. $^{9,10}$

The expression (3.7) is equal to the difference of (the absolute values of) electric charges of electron and proton and is experimentally measurable quantity. Current experimental bound puts $\kappa<10^{-21}$ [20]. If $\mathrm{SM}$ is embedded into a higher-dimensional anomaly-free theory, a priori one has no reason to expect $\kappa=0$ and the fact that its value is so small

\footnotetext{
${ }^{8}$ The gravitational anomaly (triangular diagram with one hypercurrent and two gravitons) is proportional to the trace over all hypercharges $\operatorname{Tr}[Y]$ and does not arise if one adds a right-handed neutrino: $\operatorname{Tr}[Y]=0$.

${ }^{9}$ Experimental restrictions for $\kappa_{l}$ come from the measurements of the electric charge of neutrino: $Q_{\nu} / Q_{e}=$ $\kappa_{l} \sim 10^{-15}$, while the estimates for $\kappa_{q}$ are due to the measurements of the charge of the neutron: $Q_{n} / Q_{e}=$ $3 \kappa_{q} \sim 10^{-20}[19]$.

${ }^{10}$ One should check whether the electric charge of neutrino can lead to observable effects, e.g. pair production on neutrinos in the strong electric field. Probability of such production is given by: $P_{\nu} \sim \exp \left(-\frac{\pi m_{\nu}^{2}}{Q_{\nu} E}\right)$. For the maximum electric field $E \sim 10^{7} \mathrm{Volt} / \mathrm{cm}$, taking electric charge to be $Q_{\nu}=10^{-15} Q_{e}$ and mass $m_{\nu} \sim 1 \mathrm{eV}$, we get $P \sim e^{-10^{7}}$. The effect of neutrino production becomes visible only if mass of neutrino is $m_{\nu} \lesssim 10^{-3} \mathrm{eV}$
} 
experimentally becomes a fine-tuning, which a realistic brane-world model should be able to explain.

Recall that $U(1)_{Y}$ and $S U(2)$ fields, entering (3.4) and (3.6), are the fields above the electroweak symmetry breaking scale. At lower energies it is convenient to re-express these anomalies in terms of of electro-magnetic field $\gamma_{\mu}$ and neutral field $Z_{\mu}$, which can be obtained from the $U(1)$ and $S U(2)$ fields after rotation by the Weinberg angle $\theta_{w} .{ }^{11}$ The electromagnetic current $j_{\gamma}^{\mu}=j_{\mathrm{Y}}^{\mu}+j_{3}^{\mu}$ and neutral current $j_{\mathrm{Z}}^{\mu}=-\cot \theta_{w} j_{\mathrm{Y}}^{\mu}+\tan \theta_{w} j_{3}^{\mu}$, where $j_{3}^{\mu}$ is the 3rd component of the $S U(2)$ triplet $j_{\alpha}^{\mu}, \alpha=1,2,3$. Using (3.4) and (3.6) one can easily see that (i) electro-magnetic current is conserved in the arbitrary background of electromagnetic fields (as one expected, because the electrodynamics remains vector-like in our model); (ii) there is an anomalous $\gamma \gamma Z$ coupling

$$
\partial_{\mu} j_{\mathrm{z}}^{\mu}=-\frac{2 N_{f} \kappa}{\pi^{2} \cos \theta_{w} \sin \theta_{w}} \vec{E}_{\gamma} \cdot \vec{H}_{\gamma}
$$

which implies the non-conservation of the neutral current in the parallel electric and magnetic fields ( $N_{f}$ is the number of generations); (iii) another important consequence of the presence of $\gamma \gamma \mathrm{Z}$ coupling is the non-conservation of the electro-magnetic current in the mixed electromagnetic and Z-backgrounds:

$$
\partial_{\mu} j_{\gamma}^{\mu}=-\frac{4 N_{f} \kappa}{\pi^{2} \cos \theta_{w} \sin \theta_{w}}\left(\vec{E}_{\gamma} \cdot \vec{H}_{\mathrm{z}}+\vec{E}_{\mathrm{z}} \cdot \vec{H}_{\gamma}\right)
$$

As we will see this leads to the effects similar to those, described in Section 2.2.

\subsection{Static electric field in a capacitor in a magnetic field}

Consider again a capacitor in a strong magnetic field $\vec{H}$, such that electric field $\vec{E}$, created by the capacitor, is in the same direction $x$. In such a background the current $j_{\mathrm{z}}^{\mu}$ is not conserved (3.8). This implies existence of Chern-Simons terms. These terms creates an inflow $\mathrm{Z}$ current, generating anomalous density of $\mathrm{Z}$ charge on the brane. Such a distribution of $\mathrm{Z}$ charge creates $\mathrm{Z}$ field and non-trivial $\gamma \mathrm{Z}$ background (as in the right hand side of eq. (3.9)). As a result an inflow of electro-magnetic current appears. Such an inflow creates anomalous distribution of electric charge on the brane and modifies electric field inside and outside the capacitor. Similarly to the case of electrodynamics (Section 2) we can find this static configuration of an electric field. It is given by the same expression (2.6) (see [7] for details). As a result, distribution of an electric field inside and outside the capacitor is given by expressions (2.9)-(2.11), with $m_{0}$ proportional to the magnetic field $\boldsymbol{H}_{0}$ and anomaly parameter $\kappa=\kappa_{l}+3 \kappa_{q}$ :

$$
m_{0}^{2}=\frac{N_{f} e^{2} \boldsymbol{H}_{0}}{\pi^{2} \cos \theta_{w} \sin \theta_{w}}\left(\kappa_{l}+3 \kappa_{q}\right) .
$$

\footnotetext{
${ }^{11}$ One should take into account that we are using a normalization of the gauge fields with a coupling constant in front of the kinetic term.
} 


\begin{tabular}{|c|c|c|c|c|c|c|}
\hline Model & $\kappa_{0}$ & $m_{0}, \mathrm{GeV}$ & $\tau_{0}, \mathrm{sec}$ & $L_{0}, \mathrm{~cm}$ & $\boldsymbol{E}_{\text {outside }} / \boldsymbol{E}_{0}$ & $\boldsymbol{E}_{\text {outside }}, \mathrm{Volt} / \mathrm{m}$ \\
\hline \hline new generation & 1 & $10^{-8}$ & $3 \times 10^{-16}$ & $10^{-5}$ & 0 & 0 \\
\hline charge $\nu$ & $10^{-15}$ & $4 \times 10^{-16}$ & $10^{-8}$ & $3 \times 10^{2}$ & 0.5 & $5 \times 10^{6}$ \\
\hline electric neutrality & $10^{-21}$ & $4 \times 10^{-19}$ & $10^{-5}$ & $3 \times 10^{5}$ & $6 \times 10^{-4}$ & $6 \times 10^{3}$ \\
\hline mass $\gamma$ & $10^{-36}$ & $10^{-26}$ & $3 \times 10^{2}$ & $10^{13}$ & $10^{-11}$ & $6 \times 10^{-4}$ \\
\hline
\end{tabular}

Table 2: In this Table we gather the characteristic values of the time $\tau_{0}=\frac{1}{m_{0}}$ over which the static state (2.9)-(2.10) is reached. The static electric field $\boldsymbol{E}_{\text {outside }}$ is almost constant for the distances much smaller than $L_{0}=\frac{1}{m_{0}}$ outside the plates of a capacitor. A value of the non-anomalous electric field $\boldsymbol{E}_{0}$ is taken to be $10^{7} \mathrm{Volt} / \mathrm{m}, \boldsymbol{H}_{0} \sim 10^{5}$ Gauss and the distance between the plates $d=10^{2} \mathrm{~cm}$.

Notice, that this result does not depend on either mass of the fifth dimension $M$ or on the mass of the Z-boson $\mathbf{m}_{\mathrm{Z}}$ (for $\mathbf{m}_{\mathrm{Z}} \ll M$ ). This can be understood as follows: anomaly inflow of the Z-current creates density of $\mathrm{Z}$ charge. The latter, in turn, creates electric $\mathrm{Z}$ field in the 5 th direction. This field is, of course, decaying on the scales larger than $\frac{1}{\mathbf{m}_{z}}$. However the inflow comes from the region (2.8) which is much smaller than $\frac{1}{\mathbf{m}_{z}}$. As a result, in the leading order in $\frac{\mathbf{m}_{Z}}{M}$ mass of the $\mathrm{Z}$ boson does not modify the effect.

\section{Experimental detection of anomaly inflow from extra dimensions}

Next, we propose an experiment one can conduct to determine the anomaly inflow and thus the presence of extra dimensions. As we have already seen in the previous sections, regardless of the model we are using, there is a common effect: distribution of an electric field in a capacitor changes, if the capacitor is placed in a parallel magnetic field. In particular, the electric field would appear outside the plates of the ideal capacitor with the infinite plates. A detection of such a field serves as a signature of extra dimension(s) and provides a direct measurement of the parameter $\kappa_{0}$. We should stress once again that this is a low-energy signature of extra dimensions.

We remind, that in this paper we discuss an (idealized) capacitor: two charged plates, parallel to each other and separated by the distance $d$ much less than their characteristic sizes. Such plates create an electric field $\boldsymbol{E}_{0}$ between themselves. The capacitor is placed into a magnetic field $\boldsymbol{H}_{0}$, parallel to its electric field. As an estimate we will take $\boldsymbol{H}_{0} \sim 10^{4}$ Gauss (equivalently, 10 Tesla) - which is of the order of highest stable magnetic fields, achievable in laboratory (see e.g. [21]).

To find out whether the described effect can be observed, we should estimate the value of $\kappa_{0}$. There are several quantities, which can be directly measured in experiments and whose non-zero values can serve as potential anomaly mismatch parameters. They include: the charge of the neutrino, the charge difference between the electron and the proton, etc.

New generation. One of the possible ways to make the electroweak theory anomalous, would be to have new generation which have only leptons or only quarks in them. Such a 
generation could be very massive (say, above electroweak scale) and we would not see its presence in high-energy experiments. In such a theory $\kappa_{0} \sim 1$. According to eq. (3.10) for the value of magnetic field $\boldsymbol{H}_{0} \sim 10^{4}$ Gauss one would get $m_{0} \sim 10 \mathrm{eV}$ (see the first row in Table 2). Taking distance between the plates $d \sim 1 \mathrm{~cm}$ we get, according to eqs. (2.9)-(2.10) that the electric field inside the capacitor will be exponentially (as $e^{-10^{5}}$ ) close to zero. Thus, if such a generation existed, the configuration with non-zero $\vec{E} \cdot \vec{H}$ would be forbidden. The characteristic time for reaching such a final state $\tau \sim \frac{1}{m_{0}}$ would be of the order $10^{-16}$ sec. Thus, we see that existence of such a generation can be considered experimentally excluded, as its presence would prohibit existence of parallel electric and magnetic field. This result is independent on neither energy scale of an extra dimension, nor the mass of this additional generation.

Charged neutrino. Another possibility for anomalous theory would be to add a small electric charge to the left-handed neutrino: $e_{\nu}=\kappa_{0, \nu} e$. This would make the electrodynamics anomalous. We add a mass term $\lambda \chi \bar{\nu}^{c} \nu$, which does not require a charged Higgs field and thus in such a model photon remains massless. The modern restrictions on the charge of the neutrino then give [19] $\kappa_{0, \nu}<10^{-15}$. Again, substituting this $\kappa_{0, \nu}$ into eq. (2.5), we see that after the time of the order $10^{-8}$ second the system would reach a state, in which an electric field, as measured outside the capacitor, close to the plates (closer than $L_{0} \sim 10^{2} \mathrm{~cm}$, see line 2 in the Table 2), will be of the same order as an electric field inside. Thus, this effect is very strong and, if present, can easily be detected and serve as a signature of extra dimensions.

Electric neutrality of matter. As discussed in Section 3 in SM current restriction on an anomaly mismatch parameter comes from the measured bound between electric charges of electron and proton, which gives [20] $\kappa_{0, \mathrm{EW}}<10^{-21}$. As one can see from the third row of the Table 2, an electric field outside the capacitor (2.11) would be 4 orders of magnitude lower from its initial value inside. The field inside will be lowered by approximately the same amount (according to eq. (2.9)). Whether such a change in electric field can be determined, depends on the actual setup of the experiment. If we estimate the value of $\boldsymbol{E}_{0}$ by $\sim 10^{7}$ Volt/meter, ${ }^{12}$ then an electric field outside the capacitor will be of the order $10^{4}$ Volt/meter and can easily be measured.

Massive electrodynamics. A modification of electrodynamics in which left and right moving particles have different charges is another example of anomalous theory. If left and right moving particles have different charges, they can acquire mass only via the Higgs mechanism. Since the mass term $f \bar{\psi}_{L} \psi_{R} \phi+c . c$. is gauge invariant, the Higgs field should also be charged with the charge $e_{\phi}=e_{L}-e_{R}$. The charge of the Higgs particle is proportional to $\kappa_{0}\left(e_{\phi} \sim e \kappa_{0}\right)$ and is very small. The experimental restrictions on such millicharged particles allow for their existence in the mass range from electron-volts up to hundreds of $\mathrm{GeV}$ (see, e.g., [23]). The presence of the charged Higgs field in turn implies that the photon acquires mass $m_{\gamma}^{2}=\langle\phi\rangle^{2}\left(e_{L}-e_{R}\right)^{2}$. The most stringent experimental restrictions on the mass of the photon is $m_{\gamma}<3 \times 10^{-27} \mathrm{eV}$ [24]. Taking an estimate for the Higgs's VEV to be of the order $1 \mathrm{GeV}$ (this value should be much larger than the mass of the fermions), we find the restrictions for the anomaly parameter to be $\kappa_{0, \gamma}<10^{-36}$. From the last row in Table 2 we

\footnotetext{
${ }^{12}$ Electric fields of this order are used in linear accelerators, such as the one in Stanford Linear Accelerator Center [22].
} 
see that the effect would be much smaller than in previous cases and hard to determine. ${ }^{13}$

Restriction on anomaly mismatch coefficient from atomic measurements. In the experiment described above we had macroscopic configuration with non-zero $\vec{E} \cdot \vec{H}$, created by a capacitor placed in a solenoid. On the other hand, any charged particles with spin creates a field configuration with non-zero $\vec{E} \cdot \vec{H}$. To estimate this effect, we start from the quasi-classical expressions for electric and magnetic fields, created by a particle with an electric charge $e$ and magnetic moment $\mu=\frac{e}{m}$

$$
\vec{E}=e \frac{\vec{r}}{r^{3}} ; \quad \vec{H}=\frac{3(\vec{\mu} \cdot \vec{r}) \vec{r}-r^{2} \vec{\mu}}{r^{5}} \Longrightarrow \vec{E} \cdot \vec{H}=\frac{2(\vec{\mu} \cdot \vec{r}) e}{r^{6}}
$$

Thus, in the region of the space where expression (4.1) is non-zero, inflow current (analogous to that of Section 2.2) creates non-zero anomalous charge density $\rho$. As a result, an electric field configuration will be significantly modified from the usual Coulomb to Yukawa form [7] at the distances large than $m_{0}^{-1}$, with $m_{0}$ given by an analog of formula (2.5) for $H \sim \mathrm{em}^{2}$ :

$$
m_{0} \sim m \sqrt{\kappa_{0} e^{3}}
$$

This means that the electric charge of the particle gets completely screened and the total amount of an anomalous charge which appeared on the brane is equal to the charge of the particle. Such modification of electric field could influence, for example, atomic measurements. This provides a restriction on anomaly mismatch coefficient $\kappa_{0}$ : the characteristic scale $m_{0}^{-1}$ over which the electric field changes, should be much bigger than the atomic scale $r_{a} \sim \frac{1}{e^{2} m}$. In particular, their ratio: $r_{a} m_{0}=\sqrt{\frac{\kappa_{0}}{e}}$ should be smaller than the precision with which the fine structure constant is measured (which is currently $10^{-10}$ [19]). From Table 2 one can see that this restriction allows for $\kappa_{0} \lesssim 10^{-21}$, excluding first two rows in Table 2 , but allowing for the two last ones (see also footnote 13 on this page).

\section{$5 \quad$ Initial stage of anomaly inflow}

One can ask what happens on the time scales much smaller than $\tau_{0}$, specified in the Table 2. This question was analyzed in [7]. Below we sketch the results and discuss whether they have any experimental significance.

At initial stage of the inflow one can treat it by perturbation theory in $\kappa_{0}$ and consider linear in time response. In case of the capacitor, the answer is qualitatively clear: electric charge would appear inside of the capacitor (in the region where $\overrightarrow{\boldsymbol{E}} \cdot \overrightarrow{\boldsymbol{H}} \neq 0$ initially) and changes of electric field will be of the order $\Delta \boldsymbol{E} \sim \frac{t}{\tau_{0}} \boldsymbol{E}_{0}$. The exact computation in electrodynamics show that the actual answer for an electric field, appearing outside the capacitor for times $t \ll \tau_{0}$ is: $\Delta \boldsymbol{E}=\frac{t}{\tau_{0}\left(m_{0} d\right)} \mathbf{E}_{0}$. This means, that if one did not observe any effect over the time $t_{\text {exp }}$, this time provides a lower bound on the characteristic time $\tau_{0}$ and hence on the anomaly mismatch coefficient.

\footnotetext{
${ }^{13}$ It was argued recently in [25] that interpretation of experiments leading to this bound (as well as to the less stringent one $\left(6 \times 10^{-17} \mathrm{eV}\right)$, cited in [19]) may be wrong. The new bound would be $m_{\gamma} \lesssim 10^{-14} \mathrm{eV}$. This would provide an estimate for $\kappa_{0}$ similar to that, coming from electric neutrality of the matter.
} 
As we have already mentioned above, any charged particles with spin creates a field configuration with non-zero $\vec{E} \cdot \vec{H}(4.1)$. The anomaly inflow in this case leads to an appearance of an anomalous dipole moment of the particle. Indeed, in the region of the space where expression (4.1) is non-zero, inflow current (analogous to that of Section 2.2) creates non-zero anomalous charge density $\rho$. Such density will be positive inside one hemisphere, surrounding a particle, and negative in another one. As a result, the total charge of the particle does not change. However due to the inflow any particle acquires an anomalous electric dipole moment: $d_{\mathrm{ANOM}} \sim e\left(\rho r_{c}^{3}\right) r_{c}$. Here $r_{c}$ is a Compton radius of the particle $r_{c} \sim \frac{1}{m}$ ( $m$ being the mass of a particle). Anomalous charge density grows linearly in time, being $\rho_{\text {ANOM }} \sim t E H$, with electric and magnetic fields estimated by $E \sim \frac{e}{r_{c}^{2}}=e m^{2}$ and $H \sim \frac{e}{m r_{c}^{3}}=e m^{2}$. Substituting all these values into expression for $d_{\mathrm{ANOM}}$ we get:

$$
d_{\mathrm{ANOM}} \sim e^{3} \kappa_{0} t
$$

i.e. as a consequence of anomaly inflow a particle acquires a dipole moment, which has the absolute value growing with time. A similar effect exists in SM. Namely, a background with non-zero $\vec{E}_{\gamma} \cdot \vec{H}_{\mathrm{z}}+\vec{E}_{\mathrm{z}} \cdot \vec{H}_{\gamma}$ can be created around any particle which has a spin and also electric and $z$-charges. The dipole moment there can be estimated as

$$
d_{\mathrm{ANOM}} \sim \frac{e^{2} \kappa_{0}}{\cos \theta_{w} \sin \theta_{w}}\left(\frac{m}{\mathbf{m}_{\mathrm{Z}}}\right)^{2} t,
$$

(where $\theta_{w}$ is the Weinberg angle). We see, that unlike the final stage configuration, this result depends on $\mathbf{m}_{\mathrm{Z}}$.

These results hold only in a perturbation theory, valid for times $t \ll \frac{1}{m_{0}}$, where $m_{0}$ is given by (4.2). For times, much bigger that this characteristic time, field configuration around the particle approaches static solution. Static equations are symmetric with respect to inversion $\vec{x} \rightarrow-\vec{x}$, therefore, there cannot be any dipole moment for $t \gg \tau_{\text {particle }}$.

Can an anomalous dipole moments of a particle be observed? Linear stage of anomaly inflow has a characteristic time

$$
\tau_{\text {particle }} \sim \frac{1}{m \sqrt{\kappa_{0} e^{3}}}
$$

(where $m$ is the mass of a particle). For a particle to have a dipole moment, this time should be much greater than the particle observation time. Taking as an estimate for the $\tau_{\text {particle }}$ for the electron the age of the Universe $\left(1.3 \times 10^{10}\right.$ years $)$ we get

$$
\kappa_{0}<\frac{1}{m^{2} \tau_{e}^{2} e^{3}} \sim 10^{-69} .
$$

Substituting this value into the expression for dipole moment (5.2) we obtain dipole moment $d \sim 10^{-54} e \cdot \mathrm{cm}$ which is many orders of magnitude below the currently observed experimental value $d_{e}<10^{-27} e \cdot \mathrm{cm}[19]$. Similar computation for the proton would give $\kappa_{0}<10^{-76}$ and thus even smaller dipole moment (the currently observed value $d_{p}<10^{-23} e \cdot \mathrm{cm}$ ).

As a different example let us consider restrictions on the dipole moment of a muon. In this case we can take muon life-time $\tau_{\mu} \sim 2 \times 10^{-6}$ sec as an estimate for the characteristic 
time (5.3). This gives $\kappa_{0}<10^{-35}$. However, the dipole moment, computed via (5.2) is of the order $d \sim 10^{-35} e \cdot \mathrm{cm}$ which is still many orders of magnitude smaller than the experimental data for muon $d_{\mu}<3 \times 10^{-19} e \mathrm{~cm}$.

We could take a different point of view: using eqs. (5.3) and (5.2) we can compute $\kappa_{0}$ necessary to obtain an observable dipole moment $d_{p} \sim 10^{-23} \mathrm{e} \cdot \mathrm{cm}$ for, say, proton. This gives us $\kappa_{0} \sim 10^{-4}$. However, the results of Section 4 show, that such a $\kappa_{0}$ would lead to the complete screening of an electric field in the capacitor in a magnetic field over the time $\tau_{0} \sim 10^{-14}$ sec. Therefore, such possibility can be considered experimentally excluded.

Thus, we see that anomalous dipole moment arising at the linear stage of anomaly inflow of the particle cannot be detected regardless of the value of anomaly mismatch coefficient. Although the value of anomalous dipole moment is directly proportional to the $\sqrt{\kappa_{0}}$, the time of the linear stage is inversely proportional to the $\kappa_{0}$. Thus, for the values of $\kappa_{0}$ providing dipole moments large enough to be measured, duration of the linear stage of anomaly inflow is too short to conduct an experiment.

\section{Conclusion}

In this paper we suggested an experiment which can serve as a signature of the presence of extra dimensions. This experiment is based on the observation [7] that in an anomalous theory, embedded into a higher-dimensional one, an electric charge placed in a magnetic field gets screened with the radius of the screening proportional to the anomaly coefficient in a theory. We should stress that such an effect would not be present in a purely 4-dimensional theory. Outcome of proposed experiment does not depend on the scale of extra dimensions.

Modern experimental data shows that our world is non-anomalous with a very high precision (experiments include electric neutrality of the matter, absence of the photon mass, etc.). However, we have suggested a setup in which the effect can be pronounced enough to be detected even if current experimental restrictions on the anomaly parameters are satisfied.

The negative result would serve as a restriction on such parameters as charge of neutrino and electric neutrality of the matter. However, this statement is true only in the class of models with extra dimensions, similar to the ones, described here.

Another effect of anomaly inflow is an appearance of a time-dependent dipole moment of a particle. Although we estimated that such an effect cannot be observed directly, it would be interesting to see whether its presence can have a signature in various high-energy processes. Certainly, there may exist other low-energy (and high-energy) signatures of anomaly inflow. Some of them may provide stronger restrictions on anomaly parameters and better experimental setup than the ones, discussed above. We plan to address these issues elsewhere. 


\section{Acknowledgments}

We thank I. Antoniadis, S. Dubovsky, J. Harvey, S. Khlebnikov for useful comments. The work of A.B. and M.S. was supported by the Swiss Science Foundation. O.R. would like to acknowledge the partial support of the European Research Training Network "ForcesUniverse" (contract 005104) and warm hospitality of Ecole Polytechnique Fédérale de Lausanne where a part of this work was done. A.B. would like to acknowledge the hospitality of Institut des Hautes Études Scientifiques.

\section{References}

[1] N. Arkani-Hamed, S. Dimopoulos and G. R. Dvali, "The hierarchy problem and new dimensions at a millimeter," Phys. Lett. B 429, 263 (1998). [arXiv:hep-ph/9803315].

[2] C. Bouchiat, J. Iliopoulos and P. Meyer, "An Anomaly Free Version Of Weinberg's Model," Phys. Lett. B 38, 519 (1972);

H. Georgi and S. L. Glashow, "Gauge Theories Without Anomalies," Phys. Rev. D 6, 429 (1972).

[3] S. Randjbar-Daemi and M. Shaposhnikov, "QED from six-dimensional vortex and gauge anomalies," JHEP 0304 (2003) 016 [arXiv:hep-th/0303247].

[4] L. D. Faddeev and S. L. Shatashvili, "Algebraic And Hamiltonian Methods In The Theory Of Nonabelian Anomalies," Theor. Math. Phys. 60, 770 (1985) [Teor. Mat. Fiz. 60, 206 (1984)];

C. G. Callan and J. A. Harvey, "Anomalies And Fermion Zero Modes On Strings And Domain Walls," Nucl. Phys. B 250, 427 (1985).

[5] J. A. Harvey and O. Ruchayskiy, "The local structure of anomaly inflow," JHEP 0106, 044 (2001) [arXiv:hep-th/0007037] ;

A. Boyarsky, J. A. Harvey and O. Ruchayskiy, "A toy model of the M5-brane: Anomalies of monopole strings in five dimensions," Annals Phys. 301, 1 (2002) [arXiv:hepth/0203154].

[6] D. Freed, J. A. Harvey, R. Minasian and G. Moore, "Gravitational anomaly cancellation for M-theory fivebranes," Adv. Theor. Math. Phys. 2, 601 (1998) [arXiv:hepth/9803205];

A. Boyarsky and B. Kulik, "A note on the M5 brane anomaly," Phys. Lett. B 516, 171 (2001) [arXiv:hep-th/0107041] .

[7] A. Boyarsky, O. Ruchayskiy and M. Shaposhnikov, "Observational manifestations of anomaly inflow," Phys. Rev. D 72, 085011 (2005) [arXiv:hep-th/0507098].

[8] S. Khlebnikov and M. Shaposhnikov, "Brane-worlds and theta-vacua," Phys. Rev. D 71, 104024 (2005) [arXiv:hep-th/0412306] . 
[9] V. A. Rubakov and M. E. Shaposhnikov, "Do We Live Inside A Domain Wall?," Phys. Lett. B 125, 136 (1983);

K. Akama, "An Early Proposal Of 'Brane World'," Lect. Notes Phys. 176, 267 (1982) [arXiv:hep-th/0001113] .

[10] I. Oda, "Localization of matters on a string-like defect," Phys. Lett. B 496, 113 (2000) [arXiv:hep-th/0006203] .

[11] S. L. Dubovsky, V. A. Rubakov and P. G. Tinyakov, "Is the electric charge conserved in brane world?," JHEP 0008, 041 (2000) [arXiv:hep-ph/0007179] .

[12] M. E. Shaposhnikov and P. Tinyakov, "Extra dimensions as an alternative to Higgs mechanism?," Phys. Lett. B 515, 442 (2001) [arXiv:hep-th/0102161] .

[13] A. Boyarsky, O. Ruchayskiy, M. Shaposhnikov, "Gauge fields in brane-world models," to appear.

[14] G. R. Dvali, G. Gabadadze and M. A. Shifman, "(Quasi)localized gauge field on a brane: Dissipating cosmic radiation to extra dimensions?," Phys. Lett. B 497, 271 (2001) [arXiv:hep-th/0010071] .

[15] S. L. Adler, "Axial Vector Vertex In Spinor Electrodynamics," Phys. Rev. 177, 2426 (1969);

J. S. Bell and R. Jackiw, "A Pcac Puzzle: Pi0 $\rightarrow$ Gamma Gamma In The Sigma Model," Nuovo Cim. A 60, 47 (1969).

[16] D. J. Gross and R. Jackiw, "Effect Of Anomalies On Quasirenormalizable Theories," Phys. Rev. D 6, 477 (1972);

G. 't Hooft, "Symmetry Breaking Through Bell-Jackiw Anomalies," Phys. Rev. Lett. 37, 8 (1976).

[17] A. N. Redlich, "Gauge Noninvariance And Parity Nonconservation Of ThreeDimensional Fermions," Phys. Rev. Lett. 52, 18 (1984); "Parity Violation And Gauge Noninvariance Of The Effective Gauge Field Action In Three-Dimensions," Phys. Rev. D 29 (1984) 2366.

[18] W. A. Bardeen and B. Zumino, "Consistent And Covariant Anomalies In Gauge And Gravitational Theories," Nucl. Phys. B 244, 421 (1984); S. Naculich, "Axionic Strings: Covariant Anomalies And Bosonization Of Chiral Zero Modes," Nucl. Phys. B 296, 837 (1988).

[19] S. Eidelman et al. Particle Data Group, "Review of Particle Physics," Phys. Lett. B 592, 1 (2004). PDG WWW page.

[20] M. Marinelli and G. Morpurgo, "The Electric Neutrality Of Matter: A Summary," Phys. Lett. B 137, 439 (1984).

[21] Magnet science and technology project in National Highest Magnetic Fields Laboratory (http://www.magnet.fsu.edu); 
[22] Stanford Linear Accelerator Center. See also TESLA: The Superconducting ElectronPositron Linear Collider. Technical Design Report, Part II.3

[23] M. I. Dobroliubov and A. Y. Ignatiev, "Millicharged Particles," Phys. Rev. Lett. 65, 679 (1990); "Do millicharged particles exist?," Mod. Phys. Lett. A 8, 917 (1993);

S. Davidson, S. Hannestad and G. Raffelt, "Updated bounds on milli-charged particles," JHEP 0005, 003 (2000) [arXiv:hep-ph/0001179];

M. L. Perl, P. C. Kim, V. Halyo, E. R. Lee, I. T. Lee, D. Loomba and K. S. Lackner, "The search for stable, massive, elementary particles," Int. J. Mod. Phys. A 16, 2137 (2001); [arXiv:hep-ex/0102033];

S. L. Dubovsky, D. S. Gorbunov and G. I. Rubtsov, "Narrowing the window for millicharged particles by CMB anisotropy," JETP Lett. 79, 1 (2004) [Pisma Zh. Eksp. Teor. Fiz. 79, 3 (2004)] [arXiv:hep-ph/0311189].

[24] G. V. Chibisov, "Astrophysical Upper Limits On The Photon Rest Mass," Sov. Phys. Usp. 19, 624 (1976) [Usp. Fiz. Nauk 119, 551 (1976)].

[25] E. Adelberger, G. Dvali and A. Gruzinov, "Photon mass bound destroyed by vortices," arXiv:hep-ph/0306245. 0306245; 